ISSN 1409-2441
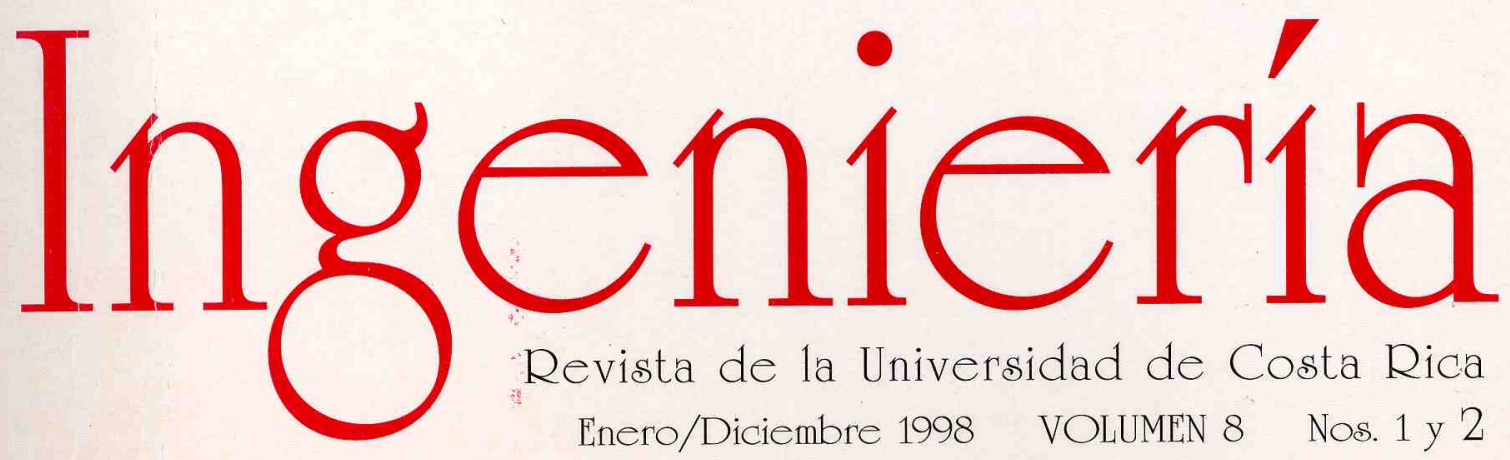

Revista de la Universidad de Costa Rica Enero/Diciembre 1998 VOLUMEN 8 Nos. 1 y 2 


\section{TRABAJOS FINALES DE GRADUACION DE LA FACULTAD DE INGENIERIA VIGENTES A 1997}

Se presentan en esta sección las Referencias Bibliográticas de los Trabajos Finales de Graduación de la Facultad de Ingeniería de la Universidad de Costa Rica correspondientes a las graduaciones del año 1997. Además del listado general, se transcriben los resúmenes de los trabajos de mayor relevancia en este período.

\section{ESCUELA CIENCIAS DE LA COMPUTACIÓN E INFORMÁTICA}

\section{Referencias Bibliográficas}

\section{Licenciatura}

Neil Thompson, Danoval, Solís del Vecchio, Alejandro y Soto Pérez, Noe A

Evaluación del rendimiento del RS/6000 de IBM segÚn las necesidades del Mercado Nacional.

Ciencias de la Computación e Informática San José, CR.

D. Neil T, A Solí s V, N. Soto P, 1997.

Madrigal Solórzano, Allan A y Villalobos U1ate, Kattia M.

Bases de Datos en WWW: Un modelo para el desarrollo en Costa Rica

Ciencias de la Computación e Informática San José, CH.

Madrigal S, K Villalobos $\{\mathbf{J}, 1997$.

Barboza C. Mauricio, Calvo Ch. Allan, y Murillo F. Gustavo.

Evaluación de un sistema administrador de bases de datos en Wl entorno distribuido según las 12 reglas de CODO y DATE para bases de datos distribuidas.

Ciencias de la Computación e Informática San José, CR.

M. Barboza C, A Calvo Ch., 1997.

\section{Maestría}

Salazar BernlÚdez, Gabriela.

Plan de aseguramiento de calidad del software para la Universidad de Costa Rica. Ciencias de la Computación e Informática San José, CR.

G. Salazar 8., 1998.

\section{Resúmenes de las tesis sobresalientes}

Barboza, M., Calvo, A. y Murillo, G. Evaluación de un Sistema Administrador de Bases de Datos en un Entorno Distribuido según las 12 reglas de Codd y Date para Bases de Datos Distribuidas. Tesis de Licenciatura, Escuela de Ciencias de la Computación e Informática, Universidad de Costa Rica, San José, Costa Rica, 1997.

La teoría sobre base de datos distribuida ha sido ampliamente tratada a través de los años, desde que ese térnlÍno surgió alrededor de la década de los 70's. Sin embargo, aun cuando se establece muy claramente el modo de operación de una base de datos distribuida, la implementación de éstas no satistace en muchos aspectos los postulados teÓricos. De hecho, aunque existen diversos productos que pretenden atender esta parte del mercado, no se sabe a ciencia cierta cuanto de la teoría ha sido implementada y en que medida la satisíacen.

Como consecuencia de esta realidad, se decide elaborar Wla investigación en la cual se selecciona un producto comercial de base de datos con características distribuidas, y se crea un prototipo basado en las características de éste. Luego, esta implementación se evalúa contra uno de los modelos más ampliamente aceptados por la categorización de una base de datos distribuida, como lo es el formulado por $\mathrm{T}$. Codd y C. J. Date, en el cual se proponen 12 reglas que un SABD debe satisfacer para ser calificado como IUl SABDD. La investigación se centrará solamente en ambientes homogéneos, por 10 cual no se llevará la misma a ambientes que incluyen diferentes plataformas de hardware, sistemas operativos, SABD's y redes, con lo que las 
reglas de la 9 ala 12 de dicho modelo, no son consideradas.

Como producto de la evaluación, se obtiene una serie de resultados, sobre los cuales se elaboran un conjunto de conclusiones acerca del alcance de la herramienta en estudio, las reglas del modelo utilizado y sobre algunas consideraciones que se deben tomar en cuenta a la hora de disefíar un ambiente de base de datos distribuidas.

Dentro del conjunto de conclusiones obtenidas se observa que el modelo de Codd y Date se mantiene aun vigente y puede aplicarse para la evaluación de cualquier SABD comercial. Sin embargo, el mismo abarca aspectos muy ambiciosos; lo cual plantea un reto importante para los investigadores del área de base de datos en los años venideros.

Adicionalmente se muestra como la versión de Informix OnLine 5J) 1 no se apega estrictamente con lo estipulado en el modelo, principalmente debido a la ausencia de un lenguaje de definición de datos apropiado, Dado lo anterior se incorporó un conjunto de tablas de configuración y LUla interfaz apropiada con la cual se logra el objetivo del modelo.

\section{Madrigal Solórzano, Allan \& Villalobos Ulate, Kattia María. Bases de Datos en WWW: Un modelo para Costa Rica. Tesis de Licenciatura, Escuela de Ciencias de la Computación e Informática, Universidad de Costa Rica, San José, Costa Rica, 1997.}

Las Bases de Datos Públicas orientadas a Web (BDPW) son sistemas que contienen infornlación de carácter públicoy que están a disposición de los usuarios de la red Internet a través del servicio World Wide Web (WWW). Para su elaboración no basta con seguir los esquemas tradicionales para el desarrollo de sistemas debido al ambiente tan dinámico en el que las BDPW se desemuelven. Esta investigación propone un modelo cuyos componentes poseen características óptimas que hacen del mismo un excelente ejemplo a segUIr: Los grupo de administración institucional y el eqmpo del Webmaster), Propósito y objetivos, Especificación de contenido, Presentación, Integración con la Base de Datos Institucional(BDI) y Modelo de la Base de datos(BD).

El seguimiento de este modelo permite obtener un sistema BDPW sencillo, tlexible, "amigable", completo y que cmnple con las expectativas de los participantes, Con el fin de facilitar este proceso, se propone el uso de una metodología basada en las actuales técnicas de desarrollo de sistemas.

Como en toda metodología, no se trata de establecer una "receta" fija e inflexible sino más bien brindar Wla "guía de trabajo" la cual está compuesta por seis etapas de complementarias y bien delimitadas que facilitan en gran medida el proceso de creación de sistemas WEB. Estas etapas representan lo que conocemos como el ciclo de vida de una BDPW, a saber: Investigación preliminar I Factibilidad, Análisis, Diseño, Implementación / Pruebas, Implantación I Promoción y Mantenimiento I Innovación.

Dos ejemplos elaborados bajo los lineamientos propuestos, son los casos de XeTOxy el Directorio Internet de Costa Rica(RACSA). Estos prototipos permitieron valorar la metodología propuesta desde dos enfoques distintos y complementarios, facilitando la elaboración de alglmas recomendaciones y conclusiones valiosas, tales como:

- Es necesario una definición clara y concreta del propósito, los objetivos, la audiencia y el contenido del WE8.

- Se debe lograr la unificación de los criterios del usuario, el analista, el diseñador y el programador.

- Conocer las fortalezas y debilidades de la herramienta facilita la labor de diseño.

- Un grupo de trabajo interdisciplinario así como buen soporte administrativo y operativo, aseguran el éxito. 
Como es de esperar, Internet continuará evolucionando, se incorporarán nuevas tecnologías y aplicaciones que brindarán mejores y más eficientes servicios a los usuarios, Los interesados en llevar a cabo proyectos de BDPW en Costa Rica, encontrarán una valiosa guía en esta investigación, que constituye un primer esfuerzo por apoyar esta labor proveyendo una metodología acorde con la evolución del medio.

Salazar Bermúdez, Gabriela. Plan de Aseguramiento de Calidad del Software para la Universidad de Costa Rica. Tesis de Maestría, Escuela de Ciencias de la Computación e Informática, Universidad de Costa Rica, San José, Costa Rica, 1998.

Este docwnento propone un Plan de Aseguramiento de Calidad que guíe el proceso de ingeniería de los sistemas computacionales en las diferentes oficinas administrativas de la Universidad de Costa Rica.•El Plan define las políticas de calidad que se deben seguir para que los productos de software desarrollados internamente o por contratación externa cumplan con los requisitos mínimos de calidad.

Para apoyar estas políticas de calidad se han elaborado un conjunto de estándares y procedimientos que permitan controlar el desarrollo del software a través de las diferentes fases del ciclo de vida de los sistemas, Los estándares que conforman el Plan de Aseguramiento de Calidad están basados en los estándares del IEEE y del IS09000, con el fin de actualizar las metodologías de desarrollo utilizadas actualmente en la Universidad de Costa Rica, de acuerdo con las necesidades de la organización y para garantizar las exigencias internacionales.

A través de la adaptación de estos estándares se logra mejorar un conjunto de deficiencias en las metodologías de desarrollo actuales, encontradas por medio de esta investigación. El Plan también tiene como objetivo facilitarle a la Universidad el poder cwnplir con las normativas de calidad que exige la Contraloría General de la República.
A nivel de aceptación este documento ha sido evaluado y aprobado por la Contraloría Universitaria y por un grupo de comités conformados por personal informático, auditores infonnáticos, abogados y lideres de proyectos seleccionados de las diferentes oficinas administrativas de la Universidad. Este es un aporte hacia la Universidad de Costa Rica pero está fuera de mi alcance su utilización, porque para esto, es necesario el apoyo incondicional de la Administración Superior y la puesta en marcha de iUl plan piloto que permita evaluar la aplicación de todas estas metodologías y prácticas recomendadas.

\section{ESCUELA DE INGENIERIA QUIMICA}

\section{Referencias Bibliográficas}

Chacón Chaves, Allan Rodrigo.

Estudio de eficiencia y mejoramiento de los separadores API de aguas oleaginosas de los planteles de RECOPE en la Garita de Alajuela, Barranca y el Alto de Ochomogo.

Tesis de Ingeniería Química, -San José, C.R.

AR. Chacón C, 1997.

$196 \mathrm{~h} \bullet$ il. 2 I refs, 25.

Fallas Avendaño, Karen.

Extracción de ácido Úrico por dos métodos a partir de diferentes sustratos orgánicos.

Tesis de Ingeniería Química, -San José, C.R.:

K. Fallas A, 1997.

Blanco Vega, Luis Alberto.

Diseño e implementación de un sistema de información para la Escuela de Ingeniería Química. Tesis de Ingeniería

Química, -San José, c.R.:

LA Blanco V., 1997.

$94 \mathrm{~h}$ : il21 refs, 21 .

Ugalde Núñez, Juven Mauricio,

Evaluación energética del esquema de distribución de vapor en el Ingenio Costa Rica.

Tesis de Ingeniería Química, -San José, C.R.:

J.M Ugalde N, 1997.

183 h.: il. - refs., 17. 
Leandro Castillo, Olman

Diseilo Construcción y puesta en marcha de un secador de transporte neumático para el Laboratorio de la Escuela de Ingeniería Química.

Tesis de Ingeniería Quimica, -San José, C.R:

O. Leandro C., 1997

92 h.: il. 17 refs., 22

Chaves Rodríguez, Víctor Hugo

Microencapsulación del agente anestésico clorohidrato de lidocaína mediante el método de coacervación.

Tesis de Ingeniería Química, -San José, c.R.:

V. H. Chaves R, 1997.

123 h.: il. refs. 29.

Jiménez Castro, Lisbeth

Evaluación de las actividades de reconversión industrial en producción, calidad y tecnología con énfasis en la pequeña y mediana industria de alimentos Tesis de Licenciatura en Ingeniería Química. -San José, C. R

L. Jiménez C., 1997

121 h.: 13il.-28refs,

Nelson Mora, Raymond Roy

Comparación del efecto del $\mathrm{pH}$, refino de la celulosa, granulometría del cemento Portland y caliza en la formación de espuma dentro de la mezcla de fibrocemento.

RR Nelson M, 1997.

91 h.: 20 il., 23 refs,

Hidalgo Muñoz, Ana Gabriela

Laboratorio integrado para la prevención y control de emisiones al aire por la combustión de hidrocarburos

Tesis de Ingeniería Química, San José, C.R MG. Hidalgo M, 1997

190 h.: il-73 refs.

\section{Resúmenes de las tesis sobresalientes}

Jiménez Castro, Lisbeth.

Evaluación de las actividades de reconversión industrial en producción de calidad y tecnología con énfasis en la pequeña $y$ mediana industria de alimentos.
Tesis de Licenciatura en Ingeniería

Química. -San José, C.R.:

L. Jiménez C. 199

121 h.: 13 il. -28 refs.

El objetivo de este proyecto es presentar un manual para la elaboración de IU1 programa de reconversión industria a nivel de empresa, en la pequeña y mediana industria de alimentos, con énfasis en tecnología, calidad y producción.

Para ello se realizó un estudio sobre el sector alimentario en Costa Rical su conformación y su competitividad. Tan\.bién se analizaron las experiencias de $\backslash$ tres empresas que ejecutaron un proyect6 de reconversión exitoso con el fin de presentarlo como ejemplo para 9tras industrias. Se presenta unja metodol gía para el establecimiento de un programa de Reconversión Industrial y los conocimierhos básicos que se deben de utilizar para, la aplicación de un diagnóstico en la industrria alimentaria. Se incluye además una guía ,de evaluación para un diagnóstico tecnológic9.

Esta metodología y diagnóstico se aplicarbn en una panadería ubicada en San Ramón. Se concluyó que se encuentran trabajando en condiciones aceptables, por ser la menor puntuación que obtuvo de $68.8 \%$ en aspectos relacionados con producto tenninado. $\mathrm{L} \sim \mathrm{S}$ mejores condiciones se presentan en materi'a prima con una puntuación de $84.2 \%$. Segúh análisis microbiológico de ambientes, superficies de operación y contenido d l pan, se obtuvieron buenos resultados en la carga microbiolÓgica presente (el recuento total no sobrepasó 40 UFC, excepto en uno de los casos. Se hacen recomendaciones co respecto a infraestructura, equipos y control, de calidad e higiene.

La inversión requerida para las mejoras planteadas es de C2 500000. Es importante mencionar que no se pueden generalizar patrones de mejora, ya que cada empresa debe fijar sus propios proyectos y reforzar aquellas áreas que se consideren claves para awnentar su capacidad para diseñar, producir y vender sus productos en competencia con otras empresas por esto sel presentan lineamientos y riemplos que 
pueden ser de utilidad a las empresas interesadas. L.J.c.

Reconversión industrial, competitividad, evaluación, alimentos.

Director de la Investigación: Dr. José Antonio Martínez-OrtÍz

Escuela de Ingeniería Química

Chaves Rodríguez, Víctor Bugo.

Microencapsulación del agente anestésico clorohidrato de lidocaÍna mediante el método de coacervación.

Tesis de Ingeniería Química. -San José, C.R.: V.B.Chaves R., 1998

123 h. :il. 29 refs.

Con el propósito de diseñar un sistema de dosificación controlada para el clorohidrato de lidocaína, se empleó para ello, la microencapsulación del principio activo por el método de eficacia al ser usado como anestésico local en seres hwnanos,

El diseño del sistema de dosificación controlada constó de tres etapas prácticas: una sene de pruebas preliminares que permitieron la adaptación del método de coacervación a la lidocaína; el estudio guiado del sistema de dosificación por medio de un diseño factorial $2^{3}$ que condujo a las condiciones más favorables de tabricación de las microcápsulas para la dositicación controlada de la lidocaína y finalmente, la caracterización de las curvas de dositlcación de la lidocaína desde las microesferas hacia el medio de transporte.

Se comprobó por medio de espectro fotometría UV, que las microesferas elaboradas mediante el método de coacervación contienen lidocaína en su interior y que la liberación del principio activo hacia el entorno, se da en fonna controlada. Además se apreció en el método de coacervación es económico, (un dólar por gramo de lidocaína encapsulado), pues el equipo y materiales empleados son fáciles de conseguir en cualquier laboratorio. Las curvas de dositlcación obtenidas, muestran una rápida liberación de la lidocaína en los primeros minutos y luego una velocidad decreciente, hasta liberar prácticamente toda la lidocaĺna del interior de las microesferas,
Esta velocidad se determina por el cambio de concentración global de lidocaína en el medio de transporte. Del análisis efectuado, con la guía del diseño tactorial, se estableció que las condiciones experimentales más convenientes para la fabricación de las microesferas son: emplear velocidades altas de agitación: $800 \mathrm{rpm}$ y usar como polímero de recubrimiento: gelatina a una concentración de $0.25 \mathrm{~g} / \mathrm{mI}$ en solución acuosa. Adicionalmente, se determinó un modelo matemático que simula los patrones de dositlcación, para cada ensayo del diseño experimental. El modelo es:

$$
\mathrm{kv} \text { estimado }=733.9-54.4 * \mathrm{Xv}+
$$

197.7* $X " 1-290.5 * X_{\mathrm{vc}}-226.5 * X_{\mathrm{pc}}$

Se realizó una prueba de dositlcación en plasma equino, con lo que se apreció la similitud en los patrones de dosificación en el agua y en el plasma, esto permitió afirmar que el sistema propuesto sirve para administrar la lidocaína en forma controlada en el plasma sanguíneo. Se sugiere extender el uso del sistema de dosificación controlada propuesto a otros anestésicos locales con estructura química similar a la lidocaína, como es la venzocaĺna. Se recomienda, antes de emplear este sistema de dositlcación controlada en la administración de lidocaína en pacientes, realizar un estudio de la reología de las microesferas en animales y posteriormente en el cuerpo humano. Esto con el fin de caracterizar la biodisponibilidad de la droga en el cuerpo, su etlcacia y adicionalmente, la toxicidad y biodegradabilidad de los polímeros usados como recubrimiento de las microesferas. V.R.C.R

Microencapsulación;Coacervación;

Liberación Controlada; Lidocaína,

Dr. Michael Chacón Scheidelar

Escuela de Ingeniería Qlúmica

\section{ESCUELA DE ARQUITECTURA}

\section{Referencias Bibliográficas}

Angulo Cruz, Ruth

Barrios AmÓn y Otoya: Documentación de interés arquitectónico y patromonial. Diseño de paseo peatonal: una respuesta.

Tesis de Licenciatura en Arquitectura . Universidad de Costa Rica - San José, C.R: 
Noviembre, 1997

$121 \mathrm{~h}$ : iL -40 ref

Rivera Ramírez, Miguel Alberto

Revaloración del Parque Nacional y el Paseo de las Damas

Práctica dirigida de Graduación en Arquitectura

Universidad de Costa Rica - San José, c.R.:

Marzo, 1997

123 h.: il. 54 ref

Garita Brencs, Henry A.

Centro de atención integral de la salud y la enseñanza para el cantón de Curridabat.

Provecto de Graduación (Licenciatura) en Arquitectura. Universidad de Costa Rica San José, C.R.

Diciembre, 1997

94 p.: iL- 11 ref

Abarca Vásquez, Jorge

Centro de interacción ecológico, recreativo y cultural "El Lago, CIEREL: hacia una visión significante del diseilo.

Proyecto de Graduación (Licenciatura) en Aquitectura. Universidad de Costa Rica San José, C. R. :

Diciembre, 1997

77 p.: il

8adilla Urei1a, Bernal

Sede de la maestría, el posgrado y el centro de investigación y capacitación en administración pública.

Proyecto de Graduación (Licenciatura) en Arquitectura. Univcrsidad de Costa Rica San José, C.R.:

1997.-42 p.

Araya Aguilar, Carlos Francisco

Propuesta de servicios particulares y comunales: Sector Este, Rincón Grande de Pavas

Proyecto de Graduación (Licenciatura) en Arquitectura. Universidad de Costa Rica San José, C. R.:

1997. -42 p.

Espinoza Sánchez, 1-Ioracio

Sede del Consejo Municipal para la planificación y el desarrollo urbano del área metropolitana.
Proyecto de Graduación (Licenciatura) en Arquitectura. Universidad de Costa Rica San José, C.R.:

1997.-77h.

Chaves Oviedo, Sandra

Centro penitenciario regional para la Zona Huetar Norte

Proyecto de Graduación (Licenciatura) en Arquitectura. Universidad de Costa Rica San José, C.R:

1997.-

Resúmenes de las tesis sobresalientes

Arce Jiménez, Federico; Ramirez Araya, Isidro

Nodo recreativo y centro generador para ciudad Quesada, San Carlos: Una propuesta de renovación urbana para el centro de la ciudad.

Proyecto de Graduación (Licenciatura) en Arquitectura. Universidad de Costa Rica - San José, C.R.:

1997.-76 h. i1 disquete. 33 ref.

La propuesta se basa en el diseño de un complejo de áreas recreativas y de interés turísticolinformativo, en el centro de Ciudad Quesada, San Carlos que logre solventar las crecientes necesidades que enfrenta la comunidad en las áreas relacionadas con el ocio y el turismo, además se intervendrá a nivel urbano los espacios adyacentes a los objetos de estudio.

Bolaños Vásquez, Alejandro

Renovación urbana para la ciudad d.e Puntarenas: muelle, plaza turística )' kioskos.

Proyecto de Graduación (Licenciatura) en Arquitectura. Universidad de Costa Rica - San José, C.R.: 1997.-62p.

Aquí se presenta una propuesta de renovación urbana para la Ciudad de Puntarenas, cuyo objetivo principal se orienta al diseño de un conjunto arquitectónico que pueda dar solución a las necesidades de carácter firncional, espacial y de infraestructura en el espacio entorno al muelle.

La propuesta contempla: 
a. Una propuesta de cambio de uso para la plataforma del muelle.

b, Una plaza de llegada, que pueda articular los entes que confluyen en este punto y las diversas actividades que allí se generan.

c, Un replanteamiento del actual tramo de dioscos.

El proyecto se empleará desde la estación de buses hasta los vestidores y servicios pÚblicos, interviniendo también todo el tramo del muelle.

\section{ESCUELA DE INGENIERIA AGRICOLA}

\section{Referencias bibliográficas.}

Carrillo Obando Carlos

Relaciones de equilibrio higroscópico y calibración del determinador motomco, modelo 919, para el cultivar vigna angularis (adzuki).

Tesis Ingeniería Agrícola, San José Costa Rica.

C. Carrillo O., 1997

Madrigal Sanabria José Ricardo

Diseño de wl sistema de extracción de polvo de impurezas de arroz en una planta procesadora.

Tesis Ingeniería Agrícola, San José Costa Rica.

JMadrigal S, 1997

\section{Mairena Navarro Edgar}

Análisis de la maquinaria y secuencia de operaciones empleadas para la preparación de café oro en un beneficio a gran escala en Costa Rica.

Tesis Ingeniería Agrícola, San José Costa Rica

E.Mairena N., 1997

\section{Morales Chaves Yenory}

Modelación hidrológica para el control de Inundaciones en fincas bananeras en el Valle del Sula.

Tesis Ingeniería Agrícola, San José Costa Rica

y. Morales Ch. 1997.

Orozco Solano Allan
Resistencia al Flujo de Aire Producido por el Café Pergamino y Oro.

Tesis Ingeniería Agrícola, San José Costa Rica

A. Orozco S., 1997

Picón Obando Diego Manuel

Resistencia del melón al daño mecánico en el proceso de empaque para exportación.

Tesis Ingeniería Agrícola, San José Costa Rica

D. Picón O., 1997.

Ureña Cubillo Juan Carlos

Diseño, construcción y ajuste de wl método de medición de conductividad térmica para frutas y vegetales.

Tesis Ingeniería Agrícola, San José Costa Rica

J.C. Ureña C., 1997.

Resúmenes de las tesis sobresalientes

Picón Obando, Diego Manuel

Resistencia del melón al daño mecánico en el proceso de empaque para exportación.

Tesis Ingeniería Agrícola, San José Costa Rica

D. Picón O., 1997.

Del total de melón (Cucwnis melo V.B. reticulatus) en el proceso de empaque que es de rechazo, un alto porcentaje es clasificado como tal, por el daño mecánico; debido a esto, fue necesario cuantificar la incidencia y las causas que lo provocan y proponer sugerencias para minimizarlo.

Se hizo una evaluación del manejo poscosecha, observando la metodología de cosecha y transporte a la planta empacadora, y en esta, se evaluó las etapas de manejo para la selección de la truta de acuerdo a su calidad. Se caracterizó la fruta determinando sus propiedades mecánicas y fisicas en cuatro ubicaciones en la planta empacadora (figura 3.1). Se observó la respuesta de la fruta a la compresión. Se construyó un instrumento y se registró la respuesta al impacto sobre cuatro superficies de contacto y para estas se probó la capacidad de absorción de energía por medio del acelerómetro, 
Se encontró que el daño mecánico producido por fuerzas de compresión wliformemente distribuidas, se evidencia Únicamente como área dañada externa. Se observó que el porcentaje de área dañada interna aumentó de 1.1 a $10 \%$ cuando la fuerza de aplicación varió de 75 a $100 \mathrm{Kgf} \quad$ El módulo de elasticidad del melón no tuvo diferencias significativas según su tamaño, pero fue mayor conforme awnentó el tamaño de la fruta, presentando un valor promedio de 0.45 MPA

En las pruebas de impacto se encontró que no hay IUla relación entre la fuerza de impacto y el porcentaje de área dañada externa, debido a que esta fuerza de impacto calculada no toma en cuenta el segundo impacto que se produce con la superficie que se encuentra detrás de las espw11as. Debido a esto las pruebas con el acelerómetro demostraron que las eSpLill1aSabsorben parte de la energía del impacto, siendo la espwna No-Bruze la que presentó el menor porcentaje de área dañada externa (2.4\%).

Con un umbral de daño mecámco establecido, es necesario por medio de la esfera instrwnentada determinar los plUltos en la empacadora donde existe daño mecánico y colocar espuma No-Bruze para que se absorba de mejor íorma la energía de impacto.

\section{Morales Chaves, Yenory}

Modelación hidrológica para el control de Inundaciones en fincas bananeras en el Valle del Sula.

Tesis Ingeniería Agrícola, San José Costa Rica

\section{Y. Morales Ch. 1997.}

El Valle del Sula es la región agrícola más importante en Honduras. Dicha región se ve afectada cada año por inundaciones de considerable magnitud, principalmente entre los meses de setiembre a diciembre. Los eventos de inundación afectan tanto la agricultura como a la industria, el sector servicios y las poblaciones generales.

El o jetivo principal del trabajo fue el desarrollo del modelo hidrológico de las cuencas tributarias al Valle del Sula (tanto internas como externas) que se usa en forma combinada con el modelo hidrodinámico para determinar el patrón de drenaje del Valle.

La principal limitante para el desarrollo de este trabajo ha sido la información de entrada. En general no se tienen registros adecuados de lluvia y cvaporación.

Primeramente, no se tiene lUla cantidad y distribución adecuadas de las estaciones pluviométricas y la cantidad de estaciones pluviográficas no es suficiente para desarrollar calibraciones hidrológicas suficientemente precisas. En segwldo lugar, se tienen muchos datos faltantes $y$ en algunos casos hasta ceses temporales de operación de las estaciones. Respecto a la información de caudales y niveles, se hallaron serias deficiencias en las curvas de descarga de Santiago y LaglUletas, además de variaciones en los niveles de cero de las escalas de Santiago, Guanacastales y Puente Chamelecón.

Entre los principales resultados obtenidos se tiene la calibración de los parámetros del modelo NAM de lluvia-escorrentía para las cuencas externas tributarias al Valle del Sula (cuencas altas de los ríos Ulúa y Chamelecón). Los valores de los parámetros obtenidos muestran las características de respuesta rápida de estas cuencas. Se halló que el tiempo de concentración del interflujo yel flujo sobre la superficie es menor a $12 \mathrm{~h}$. $\mathrm{Y}$ que el tiempo durante el cual los caudales de recesión dominan el flujo es de menos de $900 \mathrm{~h}$.

Una vez que se verificó que los resultados de las calibraciones eran aceptables se procedió a la etapa de simulación. Se simularon los caudales en los ríos UIÚa y Chamelecón durante el período de 1980 a 1992. Se construyeron series temporales de caudal usando los valores simulados de 1980 a 1992 y los registrados a 1992 a 1995; con esta información se hizo el análisis de eventos extremos de caudal a la entrada el Valle del Sula (flujo combinado de caudales en Santiago y Puentc Chamelecón). Se calculó la lluvia máxima esperada para diferentes períodos de retorno y se simularon los caudales producidos por las cuencas 
internas tributarias del Valle. Se simuló además el evento de/inundación respectivos; esto sirvió parp verificar que la esquematización ¡hecha en el modelo completo del sistf.ma de drenaje del Valle del Sula responde a los patrones reales observados,

El modelo hidiodinámico completo ha sido utilizado para la determinación de las característica,s básicas de diseño de las bordas y para el análisis de la operación de las estaci09Cs de bombeo, principalmente. En el futuro podría ser usado como herramienta para el diseíío de las diferentes obras de control de inundaciones y para la evaluación del impacto de estrategias alternativas de control de inundaciones dentro de la zona del Valle del Sula, lo que será de suma utilidad y beneficio para la toma de decisiones.

\section{ESCUELA DE INGENIERIA CIVIL}

\section{Referencias bibliográficas}

Arias Arias, José Antonio

Estudio comparativo de tuberías para drenajes pluviales en urbanizaciones.

Tesis Ingeniería Civil, San José, Costa Rica .LA Arias A, 1997

76 h: 32 ils. -52 refs,

Balma Echevería, Javier E.

Efecto de cargas cíclicas en estructmas de mampostería tipo cajón.

Tesis Ingeniería Civil, San José, Costa Rica J.E. Balma., 1997

88 h,: iluso 10 refs.

Barrantes Fuentes, Bernal

Operaciones del sistema $\mathrm{d} \sim$ bombeo aprovechando la tubería de conducción proveniente del embalse El Llano,

Tesis Ingeniería Civil, San José, Costa Rica B.Barrantes F. 1997

125 h: 23 illus. 20 cuadros; 23 refs,

\section{O Arsie Arn1ellin, Roberto}

Determinación de los parámetros dinámicos del edificio Instituto Nacional de Seguros a partir de registros sísmicos reales.

Tesis In eniería Civil, San José, Costa Rica R. O Arsie A, 1997
146 h: il. -18 rets.

Reynolds Alvarado, Harry

Análisis de estabilidad de taludes rocosos por proyección estereográfica

Tesis Ingeniería Civil, San José, Costa Rica R Reynolds A, 1997

244 h. 94 tigs. 27 refs.

Sandí Lizano, Franklin

Cargas cíclicas laterales aplicadas en paredes de mampostería (sistema convencional vs sistema viga de bloque).

Tesis Ingeniería Civil, San José, Costa Rica F. Sandí L, 1997

101 h: ils. -14 refs.

Sandoval Villalta, Ana Catalina

Determinación analítica del ancho efectivo del puntual de compresión de paredes de mampostería confinada;

Tesis Ingeniería Civil, San osé, Costa Rica AC. Sandoval V., 1997

99 h.: 50 iluso 16 refs.

ESCUELA

DE

INGENIEJ,tIA MECANICA

\section{Referencias Bibliográficas}

Leiva Herrera, Carlos

Diseño y construcción de prótesis externa, para rodilla pantorrilla

y tobillo

Tesis Ingeniería Mecánica-San José, Costa Rica.:

C. Leiva R, 1997

195 H.: 56 il. -145 refs.

Rodríguez Chacón, Alonso José

Diseñor de engranes rectos y he!icoidales

mediante un programa de computadora

Tesis de Ingeniería Mecánica.-San José, C.R.:

AJ.Rodríguez Ch., 1997

163 h.: 39il.-5 reís

Palacios Fábrega Juan Manuel

Diseño y programación de un sistema de información WWW,

Tesis Lic. Ingeniería Mecánica.-San José, Costa Rica

J.M. Palacios F", 1997

89 h. il.-32 refs. 
Pacheco Rodríguez Rodney.

Programa de computadora para síntesis cinemática de mecanismos planos

de cuatro barras.

Tesis Lic. Ingeniería Mecánica.-San José, Costa Rica

R. Pacheco R 1997

250 h.: 18 iL 11 refs.

Paz Jiménez, Alom Antonio

Estudio de la efIciencia de inyectores para turbinas Pelton

Tesis Lic, Ingeniería Mecánica,-San José, Costa Rica

AA Paz J, 1997.

153 h.: il.- 25 refs,

Lara Murillo, Nelson, -Vargas Vargas, Jorge V.

Rediseño del Sistema contra Incendios del Plantel

de Recope en La Garita de Alajuela.

Tesis Ingeniería Mecánica.-San José, Costa Rica

N. Lara M-J Vargas V., 1997.

201 h.: il. -8 refs.

Rosales Martínez, Giovanni

Estudio sobre la eficiencia de combustión del horno de fusión

de alimento de la empresa Aluminios

Nacionales, S. A

Tesis Ingeniería Mecánica.- San José, C. R:

G. Rosales M", 1997.

132 h.: il. -8 refs.

Cortés Delgado Danilo y Jiménez Acuña Mauricio

Diseño para la ampliación del sistema contra incendios

De las instalaciones de Atlas Industrial S.A

Tesis Ingeniería Mecánica.- San José, C.R.:

D. Cortés D. y M. Jiménez A, 1997.

87 h,: il. -11 ref

\section{Madrigal González, César}

Prácticas de Laboratorio para cursos de Ingeniería Mecánica basadas

en un motor de combustión interna que funciona bajo el ciclo de Otto,

Tesis Ingeniería Mecánica.- San José, C. R:

C. Madrigal G., 1997

89 h.: il.- 12 refs,
Reynolds Westover, Fernando

Programa para Simular el Maquinado de un Pieza

En una Máquina de Control Numérico,

Tesis Ingeniería Mecánica.- San José C,R.:

F. Reynolds W., 1997.

198h,: 27 il.

\section{Chi Ayi, Roberto}

Guía para la distribución eficiente y segura de combustibles en estaciones

de servicio.

Tesis de Licenciatura en Ingeniería Mecánica, San José, Costa Rica.

R Chi A, 1997

188.: il. -13 ref.

Corella Vargas, Manuel

Turbinas Kaplan "Metodología de cálculo asistido por wl programa en computadora".

Tesis Lic. Ingeniería Mecánica.-San José, Costa Rica

M. Corella V., 1997

$102 \mathrm{~h}$.

Ulate Rodríguez, Ronny Alejandro

Diseño y construcción de ıu dispositivo

Mecánico para colectar racimos de pejibaye,

Tesis Ingeniería Mecánica.- San José, Costa Rica

R. A Ulate R., 1997.

130 h.: 18 il: -7 refs.

\section{Resilmenes de las tesis sobresalientes}

Leiva Herrera, Carlos

Diseño y construcción de prótesis

Externa articulada, para rodilla

Pantorrilla y tobillo,

Tesis Ingeniería Mecánica.-San José, C.R.:

C, Leiva R, 1997

195R.: 56 il. -14 reís.

Las discapacidades de los costarricenses son valoradas principalmente en los centros médicos estatales, debido a los costos de las prótesis fabricadas a nivel mundial. Las personas que han sido amputadas de una de sus extremidades inferiores, son atendidas por la Caja Costarricense del Seguro Social, el Instituto de Seguros y el Centro de Rehabilitación Nacional, por medio del taller de prótesis y ortesis localizado en el hospital San Juan de Dios. En este centro se instalan 
prótesis con un costo acorde a las posibilidades económicas del paciente, limitando la funcionalidad y apariencia del mismo. La falta de prótesis adecuadas a bajo costo produce una serie de problemas sociales entre los que se pueden mencionar la no integración de los discapacitados a la población laboral del país.

El objetivo general de este proyecto es diseñar y construir una prótesis externa articulada de bajo costo que incluya rodilla pantorrilla y tobillo. Esta deberá proporcionar al paciente o usuario, las normas de seguridad mínimas durante su utilización, así como comodidad y buena apariencia.

La prÓtesis diseñada puede ser utilizada por personas con amputación en una de sus extremidades inferiores, a la altura del fémur, siempre y cuando mantenga el control del movimiento de la cadera.

Entre las principales condiciones de diseño se pueden mencionar las siguientes:

$$
\begin{aligned}
& \text { a. Estatura máxima del usuario: } \\
& 180(\mathrm{~m}) \text {, } \\
& \text { b. Peso máximo: } 100(\mathrm{Kg}) \text {. } \\
& \text { c, Actividad física: marcha } \\
& \text { normal. (1.92 mis) }
\end{aligned}
$$

El diseño se concentra en wl mecanismo que imita el movimiento de la rodilla y del tobillo.

Para la rodilla se utilizó un sistema con un solo eje la rotación por su sencillez, ya que este presenta

Menos piezas, cW11pliendo con los movimientos requeridos. $\mathrm{El}$ tobillo se diseño de manera que proporciona al usuario los movimientos necesarios, sin dejar de lado las condiciones de equilibrio y costo. El usuario que utilice la prÓtesis bajo los limites establecidos por el diseño puede tener la seguridad de que esta no va a fallar, ya que los materiales seleccionados son capaces de soportar los esfuerzos a que es sometida.

Por medio del diseño final se logra una combinación razonable entre funcionalidad y costo de fabricación.

$\mathrm{Si}$ la comparamos con una prótesis importada que proporcione al usuario los misinos beneficios ,su costo de fabricación es el $36.92 \%$, sin tomar en cuenta los gastos de diseño.

Para establecer con mayor exactitud las condiciones de diseño se debe llevar a cabo un análisis de las velocidades y aceleraciones (angulares y lineales ), del movimiento humano durante la marcha. A partir de los valores obtenidos del análisis ,se puede conocer el comportamiento de la prótesis en cada posición del movimiento.

Por medio de este tema de tesis se puede desarrollar IUla metodología adecuada para el diseño de componentes biomecánicos; impulsando a otros estudiantes a desarrollar proyectos relacionados con este tema.

\section{BIOMECANICA ; ORTOPEDIA \\ PROTESIS EXTERNA ; RODILLA ARTICULADA ; PANTORRILLA \\ TOBILLO.}

Director de la investigación : Ing. Willian Sánchez.

Unidad Académica :Escuela de Ingeniería Mecánica.

Rodríguez Chacón, Alonso .José. Diseño de Engranes Rectos y Helicoidales mediante un programa de computadora. Tesis de Ingeniería Mecánica. - San José, C.R, :

\section{A..J.Rodríguez Ch., 1997.} 163 h.: 39i1. -5refs

El presente trabajo de graduación tuvo como fin la creación de un programa de cómputo en lenguaje DELPHIA que permita el cálculo de transmisiones mediante engranes rectos y engranes helicoidales. Proporcionar a la Escuela de Ingeniería Mecánica de un recurso didáctico para el diseño rápido de engrane, justifica la realización del presente trabajo, Se desarrollaron modelos matemáticos para calcular analíticamente el factor geométrico $\mathrm{J}$ en engranes rectos $\mathrm{y}$ en engranes helicoidales. Para verificar la exactitud de los resultados obtenidos con el programa, estos se compararon con los resultados que arroja el método tradicional de la AGMA, American Gear Manufacturers Association (Asociación Americana de Fabricantes de Engranes).

Los valores obtenidos del factor geométrico $\mathrm{J}$ fueron muy similares que los datos 
reportados por la AGMA. Para engranes rectos, los valores resultaron ser más conservadores para nÚmeros de dientes bajos. Sin embargo, para engranes helicoidales, los valores del factor $\mathrm{J}$ resultaron generalmente mayores que los de AGMA, siendo bajo el porcentaje de diterencia.

Se consiguió finalmente lUl programa pequeño, de fácil uso y que reduce significativamente el tiempo de cálculo de las transmisiones por engranes rectos y helicoidales. Dos características fundamentales del programa provocan que este sea apto como una herramienta didáctica: primero, que posee un ambiente grático fácil de usar y segundo, que deja al usuario tomar todas las dccisiones requeridas para obtener las soluciones a los problemas. El programa es llamado Asistente para el Cálculo de Engranes Rectos y Helicoidales (AC.E.RH.) y pern1ite calcular los esfuerzos de tlexión , los esfuerzos de contacto entre dientes y las características geométricas, tanto del piño como de la rueda. Tres tipos básicos de problemas se pueden resolver: cuando los dientes del piñón y la rueda son conocidos, cuando se requiere diseñar para un nÚmero mínimo de dientes y cuando existe una distancia entre los centros de los engranes fija. Finalmente, se recomienda ampliar el modelo matemático para engranes helicoidales desarrollándolo a partir del plano transversal del engrane y añadir al programa una base de datos con las características y las propiedades mecánicas de los materiales.

DISEÑO DE ENGRANES RECTOS

HELICOIDALES; OELPHI

Director de la Investigación: lng. Alejandro Pacheco Molina

Escuela de Ingeniería Mecánica, Facultad de Ingeniería.

paz Jiménez, Alom Antonio.

Estudio de la eficiencia de inyectores para turbinas Pelton.

Tesis Lic. Ingeniería Mecánica.-San José, Costa Rica:

A.A. Paz J., 1997.

153 h.:il. -25 refs.

En este trabajo se profundiza en el estudio de algunos modelos de boquillas y agujas, y se compara el rendimiento de ellos. La ausencia de datos publicados impiden la construcción independiente de turbinas eficientes y ya que la producción eléctrica a pequeña escala se limita $\sim$ or el costo de los equipos que existen en el mercado, el generador datos y experiencia con respecto a la dimensión de turbinas Pelton haría más accesible su uso a mediano o largo plazo.

La escasez de material escrito que se refiere a este tema obligó a realizar lUla amplia consulta bibliográfica. En la fase de diseño se escogieron algunos modelos usando como referencia las dimensiones recomendadas. A estos modelos se les hizo un estudio teÓrico con el Método de Elementos Finitos, para determinar el grado de intluencia de los parámetros de diseño escogidos. Luego del análisis se seleccionaron los modelos experimentales a fabricar y se desarrolló un método experimental para comparar el rendimiento de los inyectores.

El análisis por el Método de Elementos Finitos permitió establecer que el ángulo medio de la convergencia (AMC) y el grado de convergencia de los ángulos (GCA) son dos parámetros intluyentes en el rendimiento de los inyectores. Se define $\mathrm{AMC}=(\mathrm{a}+\mathrm{J} 3) / 4$ y $G C A=(J 3-a) / 2$, donde a es el ángulo de la aguja y $13=54^{\circ}$ fue el que proporcionÓ el mejor rendimiento. Los datos experimentales mostraron 13 y AMC son tactores muy intluyentes en la eficiencia de un inyector, pero que los datos no fueron suficientes para determinar la intluencia del GCA Los datos obtenidos permitieron generar curvas de mejor ajuste con las cuales se estableció que el AMC óptimo y el 13 Óptimo para las condiciones de operación es AMCop $=22 Y \quad$ y $\mathrm{J} 30 \mathrm{p}=50,4^{\circ}$ respectivamente, con los cuales se puede obtener el ángulo de aguja óptimo: aop $=38,7$ ".Los valores J30p y aop se encuentran dentro de las recomendaciones dadas en la bibliografía consultada,

La tcpdencia de las curvas muestra que se pueden alcanzar mayores eficiencias con los valores de AMC menores a los obtenidos con los modelos fabricados. Para la caída 
experimental estudiada en esta turbina, las mayores eficiencias probablemente ocurran cuando $\quad \mathrm{AMC}=\left[200-24^{\circ}\right]$,por lo cual es recomendable fabricar boquillas y agujas que permitan alcanzar este ámbito. Es necesario estudiar la influencia de otras variables geométricas y parámetros de diseño que podrían hacer que los ángulos que dan mayor eficiencia fueran diferentes a los encontrados.(APJ).

TURBINAS,

PEL TON;INYECTORES;EF1CIENCIA

DE TURBINAS.

Director de investigación: Glen Dewey.

Escuela de Ingeniería Mecánica,

Pacheco Rodríguez, Rodney.

Programa de computadora para síntesis cinemática de mecanismos planos de cuatro barras.

Tesis Lic. Ing. Mecánica. -San José, C.R. R. Pacheco R., 1997.

250 h.: 18 iL - Ilr,:fs.

Se presenta en este infoffile, el desarrollo de un programa para computadora con el cual, es posible realizar síntesis de mecanismos de cuatro barras . El programa puede resolver problemas tanto de Guía de un Cuerpo Rígido como Generación de una Trayectoria, para lo cual permite especificar entre 2 y 5 posiciones múltiplemente separadas (posición, velocidad, aceleración, etc, ),El objetivo principal de este proyecto fue crear el programa para ser utilizado como una herramienta didáctica, en la enseñanza de los métodos de síntesis cinemática, en la carrera de Ingeniería Mecánica, El objetivo específico más importante, file proveer al programa con una interfaz gráfica por medio de la cual, se realizará de manera visual todo el intercambio de/información y además, fuera posible utilizárla para generar la animación de los mecariismos sintetizados,

Para llevar a cabo la elaboración del programa llamado SintMec, y con el fin de cumplir con los objetivos antes mencionados, se eligió utilizar el lenguaje de programación Delphi, el cual funciona bajo ambiente Windows. Se subdividió el programa en tres módulos: 1. Introducción de posiciones, 2. Síntesis del mecanismo, 3. Animación del mecanismo, con el fin de sugerir al usuario el proceso lógico que debe llevar a cabo siguiendo el orden de esta subdivisión. Para lograr que el programa fuera de uso intuitivo, éste fue provisto de una serie de herramientas tendientes a facilitar el trabajo del usuario.

También, al programa se le incorporó una utilidad para crear salidas impresas a escala con el fin de que el usuario pudiera realizar corroboraciones gráficas y así reforzar aún más el carácter didáctico de este.

Se concluyó al íinalizar este proyecto, que el programa realizado no solo cwnple con el objetivo de ser didácticamente útil, sino que además se le puede dar a éste un uso profesional. Se encontró también que el programa posee características importantes como: capacidad de realizar síntesis en corto tiempo, fácil de aprender, versátil en su funcionamiento, 'puede ser utilizado para la enseñanza de otros ámbitos más funda mentales de la teoría de los mecanismos, etc. También se concluyó que la síntesis de mecanismos un proceso iterativo, en el cual se descarta gran parte de las síntesis al aplicar los criterios de selección, es necesario contar con una herramienta la cual permita la creación de varias síntesis en corto tiempo, para así agilizar dicho proceso. R.P,R

SÍNTESIS; CINEMÁTICA;

MECANISMOS; PROGRAMA.

Director: Ronny Alfaro Castillo.

Escuela de Ingeniería Mecánica. 